D

${ }^{1}$ National Research Council of Italy, Institute of Biomedicine and Molecular Immunology,

Palermo, Italy.

2Dept of Economics, Business and Statistical Science, University of Palermo, Palermo, Italy.

3Dept of Paediatrics, "Sapienza" University of Rome, Rome, Italy.

\title{
From research question to dissemination: how to design, analyse and present study results
}

Cite as: Fasola S, Cilluffo G, Nenna R, et al. From research question to dissemination: how to design, analyse and present study results. Breathe 2018; 14: 232-234.

\section{Doing science}

\section{Introduction}

Today, the term "study" is very frequently used in quite a broad sense: survey, clinical trial, analysis, research and other terms with deeply different meanings are often generally indicated as "studies". A "study" can be generally defined as a process composed of several actions aimed to verify one or more hypotheses (objectives), with the ultimate goal of sharing the answers (results) with a target audience, in the form of a scientific paper, presentation or poster.

For all research studies, the key for the success of a study lies in the careful design of all its different phases, from the statement of the objectives to the dissemination of the results. The different phases of a research study may be summarised as the following three parts: 1) design, 2) running, and 3) presentation of results. The setting of the three phases may differ depending on the type and the scope of the study; in the remainder of this article, a set of general guidelines are presented within the particular context of clinical studies.

\section{Study design}

The aspiration of people working in the research field is to have their own contribution acknowledged with respect to one or more study areas. The element that initiates the study process is the idea. The term "idea" should not be confused with "discovery", but rather it should be intended as coming across one or more questions to translate into objectives. However, to produce results of high impact, the answers to such research questions should be completely or at least partially unknown. Only in this case can we say that the idea is original, and it will lead to innovative objectives and innovative studies. In practice, the best way to stimulate creativity is the deep knowledge or a deep study of the topics of interest and related scientific literature, to find out possible areas that are unexplored, partially explored, or possibly inadequately explored. However, quite often, the idea rises up suddenly or unexpectedly.

Once the idea is born, it has to be translated into objectives. At this stage, it turns out to be very 
important to listen to the opinion of other experts about our proposals, to find out possible weaknesses and strengths of our ideas. The most interesting and innovative aspects will define the primary objectives of the study, while the other ancillary aspects will define the secondary objectives.

The objectives should be specific and stated as clearly as possible, therefore delimiting our area of investigation. To further define the area of investigation and provide scientific bases to the study, it is crucial to hypothesise a possible answer to the research question, i.e. formulate a research hypothesis; in this sense, the objective of the study will simply consist of testing this hypothesis. Sometimes, the research hypothesis may have quite a broad formulation, for example "Smoking has a direct effect on the respiratory system due to swelling and narrowing of the lung airways." In this case, the general objective can be decomposed into several specific objectives, such as "Smoking has a direct effect on respiratory symptoms", "Smoking has a direct effect on the forced expiratory volume in the first second", and so on. Finally, the objective should not be too difficult or too expensive to achieve.

Once the objectives have been stated, the work plane must be defined accordingly, by establishing its phases, contents, feasibility and timing. Generally, this is accomplished by drafting a study protocol that clearly identifies the scientific background and rationale of the study, the required methodologies (study type, population, interventions, outcomes, technical equipment) and the expected results.

\section{Statistical plan}

For a proper design of a clinical study, the role of an expert biostatistician is very important. In fact, based on the research question and the primary objectives, the statistician helps to identify the most appropriate study type, the required sample size and the statistical analyses to be performed.

Concerning the study type, the biostatistician may find it appropriate to carry out a cohort study or a case-control study to test hypotheses about disease aetiology, a randomised controlled trial (possibly a multicentre trial, especially when a large sample size is required) to test hypotheses about the efficacy of an experimental drug, or a diagnostic accuracy study to test hypotheses about the predictive ability of a clinical marker.

Concerning the sample size, the statistician provides the minimum number of individuals required to minimise the probability of making interpretation errors. These interpretation errors consist of stating that the research hypothesis is true when actually it is not (by chance, the data corroborate) or, conversely, stating that the research hypothesis is not true when actually it is (by chance, the data do not corroborate it). Usually, for a correct sample size calculation, the knowledge of some preliminary information is required: the expected effect sizes, the standard deviation of an outcome, or the incidence of disease in nonexposed individuals [1]. Such information may be derived from a so-called "pilot study", or harvested from previously published similar studies.

Finally, concerning the statistical methodologies, the biostatistician identifies the most appropriate tools for descriptive analyses, tables, figures, rates, indicators, tests and mathematical models, according to the objectives and nature of the variables involved. The analyses most commonly carried out by a biostatistician are: diagnostic accuracy studies (sensibility, specificity, receiver operating characteristic (ROC) curve); comparison of variable means between groups (ANOVA), possibly with paired data; correlation studies; linear regression; logistic regression; analyses of concordance (Cohen's $\kappa$ ) and reproducibility (Bland-Altman); longitudinal data analyses (survival analysis, multistate models); multivariate analyses (cluster analysis, latent class analysis, principal component analysis); and systematic reviews.

There are several available packages to perform statistical analyses, the most known and powerful being SAS, M-PLUS, STATA, SPSS, and R. In particular, $R$ is a free and open-source software that offers users the possibility to customise their analyses, creating new functions or modifying the source code of the existing ones. Very importantly, the source code of the sessions of analysis (not only the output) can be saved to file and then easily re-executed, improved or finalised. See [2] for an introductory guide to the R environment.

\section{Running the study}

Carrying out the study requires running the actions planned in the study protocol as strictly as possible. Of course, this phase is differently characterised according to the study type, and it often requires teamwork and collaboration with experts. Continuing to consider a clinical study as an example, its implementation usually involves a recruiting phase (data collection) as scheduled, the phase of data analysis and the production of preliminary reports illustrating the results. In particular, observing unexpected results may highlight possible concerns that may have affected the process of data collection and analysis, suggesting possible ways for improving them. In this regard, several guidelines illustrate the main potential sources of bias in clinical studies [3], as detailed in table 1.

\section{Presentation of results}

For almost all studies, the final goal is to disseminate and discuss the obtained results with the public. There are two main modalities for communicating the study results: oral presentations, characterised by the production of slides (or posters) to expose 
Table 1 Main sources of bias in clinical studies

\begin{tabular}{ll}
\hline Type of bias & Description \\
\hline Selection bias & $\begin{array}{c}\text { Missing or inadequate randomisation: participant characteristics, including potential confounders, may } \\
\text { differ between the experimental arms. }\end{array}$ \\
Performance bias & $\begin{array}{l}\text { Missing or inadequate blinding of outcome assessor: a physician may be induced to detect improvements } \\
\text { in treated individuals. }\end{array}$ \\
Detection bias & $\begin{array}{l}\text { Missing or inadequate blinding of participants: an individual may be induced to perceive health } \\
\text { improvements if they know they are being treated. }\end{array}$ \\
Attrition bias & $\begin{array}{l}\text { Too many withdrawals, or unbalanced dropout rates among the arms: loss in statistical power, misleading } \\
\text { outcome interpretation. }\end{array}$ \\
Reporting bias & $\begin{array}{c}\text { Selective outcome reporting: in general, authors may be induced to select the findings to report (for } \\
\text { example, due to presence of a funding institution). }\end{array}$ \\
\hline
\end{tabular}

the results; and reports in written form, typically theses or scientific papers to submit for publication.

A "speaker" and a "writer" rely on different techniques to convey their message [4]. Unlike a "reader", a "listener" is "forced" to pay attention to everything presented, with the risk that they may have problems following the speech when they are not able to understand or simply not interested in some details. Therefore, it is crucial to correctly identify the target population and organise the speech accordingly, trying to be clear and concise and engage the listeners, possibly with funny moments. A speaker usually has a few tens of minutes available, and it is better to present a few, well-arranged slides, rich in pictures, plots, tables, flow-charts and bulleted points, rather than simply narrative next. Emotion is another aspect that may negatively affect the speaker performance in oral communications; in this regard, it may be useful to practise the presentation several times to gain security.

Due to the impossibility for a reader to directly interact with the writer (for example by asking for clarifications), written communication needs a more rigorous structure. When deciding to prepare a manuscript for submission, the first step should be identifying the authors (according to the required expertise) and an appropriate journal (according to its aims). Each journal provides its own specific guidelines for authors, but in general, the following sections are required: title page, abstract, introduction, methods, results, discussion, conclusions and references.

The title page includes the title, authors, affiliations, address for correspondence, key words and sponsors, if any. The abstract has a similar structure to the main article and provides an effective summary of the paper using no more than 250 words. The introduction (1 page) explains the rationale of the paper, highlights its innovative aspects and illustrates the aims of the study. The methods section (2-3 pages) illustrates the study design (including ethics issues), participants (inclusion and exclusion criteria), interventions, outcome and risk factor definitions, assessment procedures, sample size calculations and statistical analysis. The results section (2-3 pages and approximately six graphical items such as tables and figures) illustrates the main findings, possibly reflecting the steps in the methods section. The discussion (3-5 pages) emphasises take-home messages, comparison with previous literature, interpretation and mechanisms, clinical relevance, strengths and weaknesses. Conclusions (a few sentences, again remarking on the take-home messages) and the list of references (no more than 40) finalise the paper. See [5] for more details.

\section{Conflict of interest}

None declared.

\section{References}

1. Cohen J. Statistical Power Analysis for the Behavioral Sciences. 2nd Edn. Hillsdale, Lawrence Erlbaum Associates, 1988.

2. R Core Team. R: A Language and Environment for Statistical Computing. Vienna, R Foundation for Statistical Computing, 2017. www.R-project.org

3. Sardanelli F, Di Leo G. Biostatistics for Radiologists: Planning,
Performing, and Writing a Radiologic Study. Milan, SpringerVerlag Italia, 2009

4. Sweeney S. English for Business Communication. 2nd Edn. Cambridge, Cambridge University Press, 2003.

5. Sterk PJ, Rabe KF. The joy of writing a paper. Breathe 2008; 4 : 224-232. 